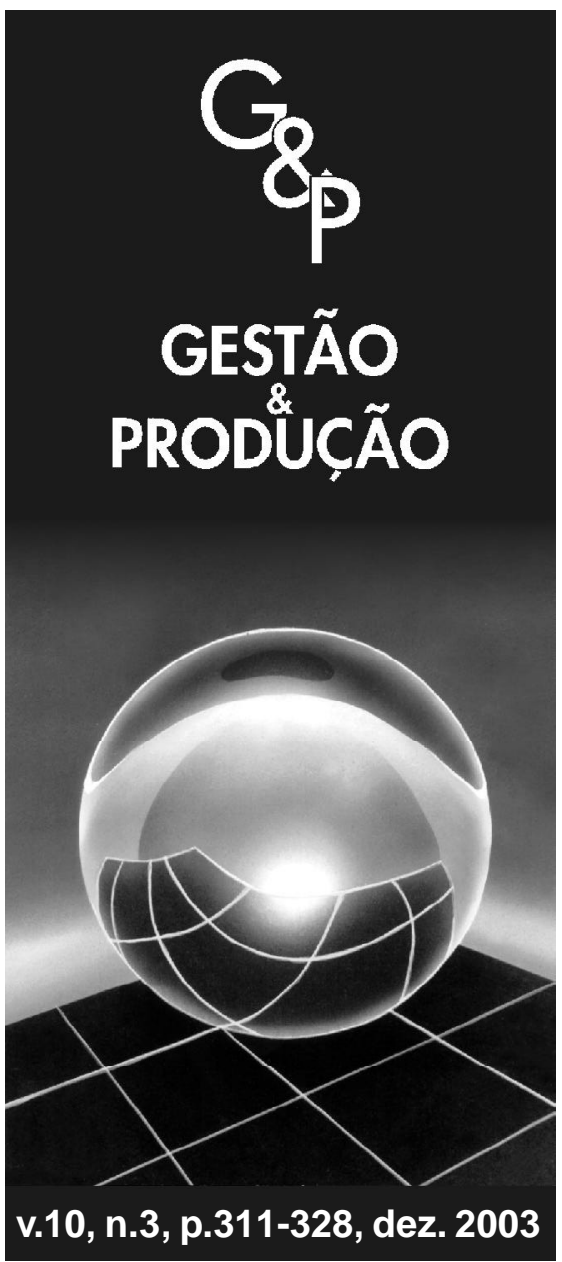

\title{
UM ESTUDO DE CASO DE GESTÃO DE PORTFOLIO DE PROJETOS DE TECNOLOGIA DA INFORMAÇÃO
}

\author{
Renato de Oliveira Moraes \\ Faculdade de Economia e Administração da Universidade de \\ São Paulo, Rua Pe. Benedito Maria Cardoso, Bl. f-10, \\ apto. 404, CEP 03169-060, São Paulo, SP, \\ e-mail: renato.moraes@perceptron.com.br
}

\section{Fernando José Barbin Laurindo}

Departamento de Engenharia de Produção da Escola Politécnica da Universidade de São Paulo, Av. Prof. Almeida Prado, 128, Tr. 2 Biênio, 2ำ andar, CEP 05508-900, São Paulo, SP, e-mail: fjblau@usp.br

Recebido em 1/7/2003 Aceito em 25/11/2003

\section{Resumo}

O papel da tecnologia da informação (TI) nas organizações pode variar de simples suporte administrativo até uma situação estratégica, em que ocupa posição hierárquica superior em organizações que disputam mercados em crescente competição. Por outro lado, ainda há discussão sobre as dificuldades de se obterem evidências do retorno dos investimentos feitos em TI. Embora haja diferentes explicações para esse fato, há certo consenso sobre o significativo crescimento dos investimentos em TI na última década. As decisões sobre quais projetos de TI devem ser implementados são, freqüentemente, determinantes do desempenho empresarial e são capazes não somente de mudar o posicionamento estratégico das organizações, mas também podem modificar a estrutura de competição do setor como um todo. Este artigo procura analisar, por meio de um estudo de caso, a seleção de projetos de TI segundo uma abordagem de gestão de portfolio, considerando os diferentes tipos de projetos, os impactos desses projetos nos fatores críticos de sucesso da empresa, os custos do projeto, bem como os riscos associados. Contempla, portanto, aspectos de eficiência (uso de recursos) e eficácia (obtenção de resultados positivos para a organização).

Palavras-chave: tecnologia da informação, gestão de portfolio de projetos, avaliação ex ante. 


\section{Introdução}

$\mathrm{O}$ papel da tecnologia da informação (TI) nas organizações pode variar de simples suporte administrativo até uma posição estratégica (Henderson \& Venkatraman, 1993; Mcfarlan, 1984). A função estratégica da TI tem ocupado posição destacada em organizações líderes que disputam mercados em crescente competição (Porter, 2001). Dois pontos básicos podem ser usados para compreendê-la: obtenção de vantagem competitiva na cadeia de valor e aumento de competências essenciais (Porter \& Millar, 1985; Duhan et al., 2001). Por outro lado, embora haja certo consenso sobre o significativo crescimento dos investimentos em TI na última década, permanece uma importante discussão sobre as dificuldades de obter evidências do retorno dos investimentos feitos em TI, tanto no âmbito da empresa individual como no agregado da economia. Essa discussão tem sido chamada de "paradoxo da produtividade" (Strassman, 1990; Brynjolfsson, 1993). Contudo, deve-se destacar que um número crescente de diferentes explicações para esse fato tem sido publicado na literatura acadêmica (Stratopoulos \& Dehning, 2000; Brynjolfsson \& Hitt, 1998).

As decisões sobre quais projetos de sistemas de informação (SI) devem ser implementados são, freqüentemente, determinantes do desempenho empresarial e são capazes não somente de mudar o posicionamento estratégico das organizações, mas também podem modificar a estrutura de competição do setor como um todo, como aconteceu nos setores financeiro e de transporte aéreo (McFarlan, 1984; Laurindo, 2002).

Entretanto, a avaliação da eficiência dos projetos de TI (em relação a prazos, custos e qualidade) ainda é uma questão problemática, por conta da incerteza e da complexidade inerentes a esse tipo de projeto. Atingir as metas de eficiência dos projetos envolve uma série de trade-offs entre seus escopos e os recursos disponíveis. Assim, os gerentes de projeto de TI utilizam um conjunto de conceitos, ferramentas e técnicas para melhorar o desempenho dos projetos, bem como a eficácia e a eficiência organizacional (Carvalho et al., 2003; Laurindo et al., 2003).

Este artigo visa a investigar a gestão de portfolio de projetos de TI, considerando a ligação desses projetos com os objetivos estratégicos da organização, os riscos associados e a utilização dos recursos.

O impacto estratégico das aplicações de TI e seus efeitos na organização e na indústria foram analisados por McFarlan (1984), por meio do Grid Estratégico, que permite visualizar a relação entre a estratégia de TI e a estratégia da organização. McFarlan (1981) também analisou a seleção de projetos de novas aplicações da TI, considerando os riscos associados a cada projeto e também os riscos da carteira de projetos. Jiang \& Klein (1999a) estudaram a seleção de tais projetos considerando empresas classificadas nas diferentes possibilidades do Grid Estratégico. Para investigar esses conceitos, a abordagem metodológica adotada para o desenvolvimento da pesquisa tratada neste artigo foi a de estudo de caso (Yin, 1991; Claver et al., 2000). A base teórica engloba conceitos de gestão de portfolio de projetos (em especial os de TI McFarlan, 1981), fatores críticos de sucesso (Rockart, 1979), impacto estratégico da TI (McFarlan, 1984), e tipologias de aplicações de TI (Farbey et al., 1995) e a questão dos riscos em projetos (Archibald, 1976; Jiang et al., 1996; Jiang \& Klein, 1999b).

Os resultados iniciais indicaram a necessidade de ferramentas que facilitassem o entendimento e a disseminação da gestão do portfolio de projetos. Este artigo pretende contribuir com uma abordagem ampla e sistemática de seleção de projetos de TI, trazendo novos pontos e alternativas para a discussão dessa questão, de importância crescente na competitividade das organizações.

\section{Fundamentos teóricos}

A revisão teórica tem início com uma visão do papel dos projetos de TI nas estratégias das organizações (Rockart, 1979; McFarlan, 1984; Farbey et al., 1995) e segue com a seleção de 
projetos (em especial os de TI-McFarlan, 1981) e a consideração dos riscos associados que afetam a probabilidade de sucesso (Archibald, 1976; Jiang et al., 1996; Jiang \& Klein, 1999b). Também é discutida a influência da situação dos projetos em andamento (Cleland \& Ireland, 2002; Maximiano, 1997) sobre a seleção de novos projetos, já que eles concorrem pelos mesmos recursos. E, por fim, há a questão do acompanhamento dos projetos em andamento (Archibald, 1976; Farid \& Karshenas, 1988; Dinsmore, 1992; Moraes, 1999).

\subsection{Projetos de tecnologia da informação e estratégia}

Nos primeiros estágios de uso de computadores nas empresas, a capacidade limitada de hardware e software restringia de forma bastante intensa as possibilidades de aplicações da TI. Assim, apenas aplicações mais estruturadas e menos complexas podiam ser implementadas, como, por exemplo, folha de pagamento, controle de estoque e contas a pagar. Com o desenvolvimento tecnológico, o leque de possibilidades foi ampliado e uma gama de novas alternativas de aplicações de TI tornouse possível. Desse modo, gerou-se um aumento da incerteza acerca da decisão sobre a seleção dos projetos a serem desenvolvidos e implementados (Laurindo et al., 2001).

Uma das primeiras propostas para o problema de seleção e priorização de projetos de TI foi o método dos Fatores Críticos de Sucesso (FCS), proposto por Rockart (1979). Esse método, que ainda é amplamente utilizado, foca, principalmente, os sistemas de informação gerenciais e executivos, e é baseado na definição, por parte dos altos executivos, das atuais necessidades representadas pelos FCS. Rockart (1979) define FCS como as áreas onde um resultado satisfatório "garante o sucesso do desempenho competitivo da organização". O autor afirma que os principais FCS podem ser identificados na estrutura do setor, na estratégia competitiva, na posição da indústria, na localização geográfica e nos fatores ambientais e temporais. Embora tenha sido concebido primariamente para definição de sistemas de informação, esse método apresenta importante impacto nas práticas gerenciais e de planejamento estratégico. É comum definir FCS para diferentes funções da empresa, como FCS de marketing, de manufatura, de projeto, dentre outros.

Porter $(1979,1996)$ destacou a importância do posicionamento da empresa em sua indústria, de forma a obter vantagens competitivas sustentáveis por meio da escolha de uma das três possíveis estratégias genéricas: liderança em custos, diferenciação e foco. Para esse autor, a competitividade de uma indústria resulta da ação de cinco forças: clientes, fornecedores, produtos substitutos, novos entrantes e a rivalidade entre os competidores atuais. Quanto mais intensas forem essas forças, maior a concorrência entre as empresas de uma indústria e, por conseguinte, menor o potencial de lucratividade.

McFarlan (1981) analisou a seleção de projetos de novas aplicações de TI considerando os riscos associados a cada projeto e também os riscos da carteira (portfolio) de projetos. Esses riscos incluem custo, prazo, desempenho técnico, superestimação dos benefícios dos SI e incompatibilidade de hardware e/ou software com o SI. O mesmo McFarlan (1984) propôs o Grid Estratégico, que permite visualizar a relação entre estratégia de TI (atual) e carteira de aplicações (futuro), definindo quatro regiões, cada qual representando um possível papel para a TI dentro da organização: Suporte, Fábrica, Transformação e Estratégico (Figura 1):

- Suporte: as aplicações presentes e futuras de TI têm pouca influência na estratégia da organização.

- Fábrica: as aplicações da TI são importantes para o sucesso da operação da empresa, mas não há nenhuma aplicação estratégica planejada para o futuro.

- Transformação: a TI está saindo de uma situação de baixa importância (região de Suporte) para assumir um papel de importância estratégica na organização, em face das novas aplicações de TI planejadas para ser implementadas no futuro próximo. 
- Estratégico: a TI é muito importante na estratégia atual do negócio e as novas aplicações planejadas manterão a importância estratégica da TI no futuro.

A fim de avaliar o impacto estratégico da TI, McFarlan analisou o efeito das aplicações de TI nas cinco forças competitivas (Porter, 1979). Havendo impacto em ao menos uma dessas forças, a aplicação de TI (ou o conjunto de aplicações de TI) poderá ser considerada estratégica.

Diferentes aplicações de TI, conforme sua natureza, abrangência e grau de modificação imposto, apresentam diferentes impactos em uma organização. Com base nessas considerações, diferentes classificações de aplicações de TI foram propostas.

Entre as diversas taxonomias de aplicações de TI, Farbey et al. (1995) propuseram uma classificação em oito diferentes tipos: mudanças obrigatórias; automação; sistemas de valor adicionado direto; sistemas de informação gerenciais (SIG) e sistemas de apoio à decisão (SAD); infra-estrutura; sistemas interorganizacionais; sistemas estratégicos; e transformação do negócio.

\subsection{Seleção de projetos de TI e a probabilidade de sucesso dos projetos}

Jiang \& Klein (1999a) estudaram a seleção de projetos de TI considerando as quatro diferentes possibilidades do Grid Estratégico. Empresas classificadas nas categorias estratégico e transformação são, normalmente, mais dispostas a assumir riscos e têm controles mais rigorosos para os projetos de TI. As empresas classificadas como suporte consideram o custo como o principal critério para seleção e condução dos projetos de TI.

A seleção de projetos de TI deve considerar, simultaneamente, aspectos relacionados à eficiência (uso dos recursos) e à eficácia (obtenção de resultados para a organização), além de analisar seus riscos. Esse critério é muito importante em mercados altamente competitivos de economia globalizada. Neste artigo, projetos de TI são considerados projetos de pesquisa e desenvolvimento (P\&D), e se usa a idéia de gestão de portfolio (carteira).

Essa abordagem permite que os gerentes tenham uma visão ampla das alternativas de projetos que podem maximizar os resultados de toda a carteira (McFarlan, 1981; Carvalho et al., 2003).

As incertezas podem ser percebidas de diferentes formas durante o desenvolvimento de um projeto. Neste artigo, serão consideradas as incertezas em relação à obtenção dos resultados esperados em termos de custo, prazo, qualidade do resultado final do projeto e seus impactos na organização.

Nota-se que, segundo Archibald (1976), as incertezas de projeto diminuem conforme o projeto avança (Figura 2).

Jiang \& Klein (1999b) e Jiang et al. (1996) associam os fatores de risco de projetos de sistemas de informação ao sucesso desses projetos.

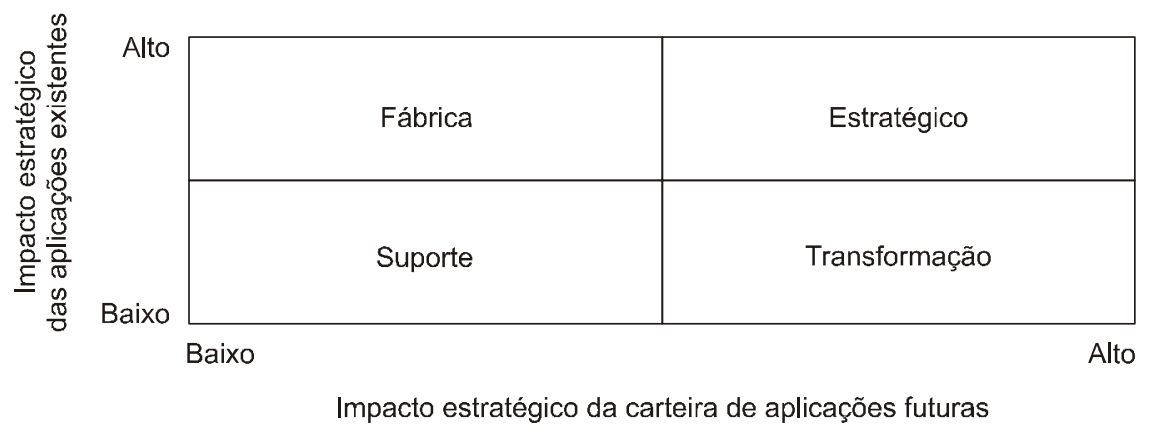

Figura 1 - Grid Estratégico do impacto das aplicações de TI (McFarlan, 1984). 
Por meio da análise de cerca de 86 projetos, foram identificados os principais fatores de risco que explicam o sucesso de projetos de sistemas de informação, a saber:

- novidade da tecnologia;

- tamanho do projeto;

- falta de conhecimento necessário da equipe como um todo;

- falta de capacidade da equipe em relação à tarefa ou ao negócio no qual o sistema irá operar;

- falta de capacidade técnica da equipe de desenvolvimento;

- falta de suporte e envolvimento do usuário no projeto;

- insuficiência de recursos;

- falta de clareza na definição de papéis e responsabilidades no projeto;

- complexidade da aplicação;

- Falta de experiência do usuário em relação ao negócio e ao trabalho em projetos.
Keil et al. (1998), ao estudarem os riscos de projetos de sistemas de informação, utilizaram a técnica DELPHI, o que levou à construção de uma lista dos fatores de risco em ordem decrescente de importância:

1. falta de apoio e comprometimento da alta gerência;

2. falha em obter o comprometimento dos usuários;

3. requisitos não entendidos corretamente;

4. falta de envolvimento adequado dos usuários;

5. falha em gerenciar as expectativas dos usuários;

6. mudanças de escopo e objetivos;

7. falta de conhecimento e habilidades necessários por parte da equipe do projeto;

8. falta de "congelamento" dos requisitos;

9. introdução de novas tecnologias;

10. recursos insuficientes ou não apropriados;

11. conflitos entre departamentos dos usuários.
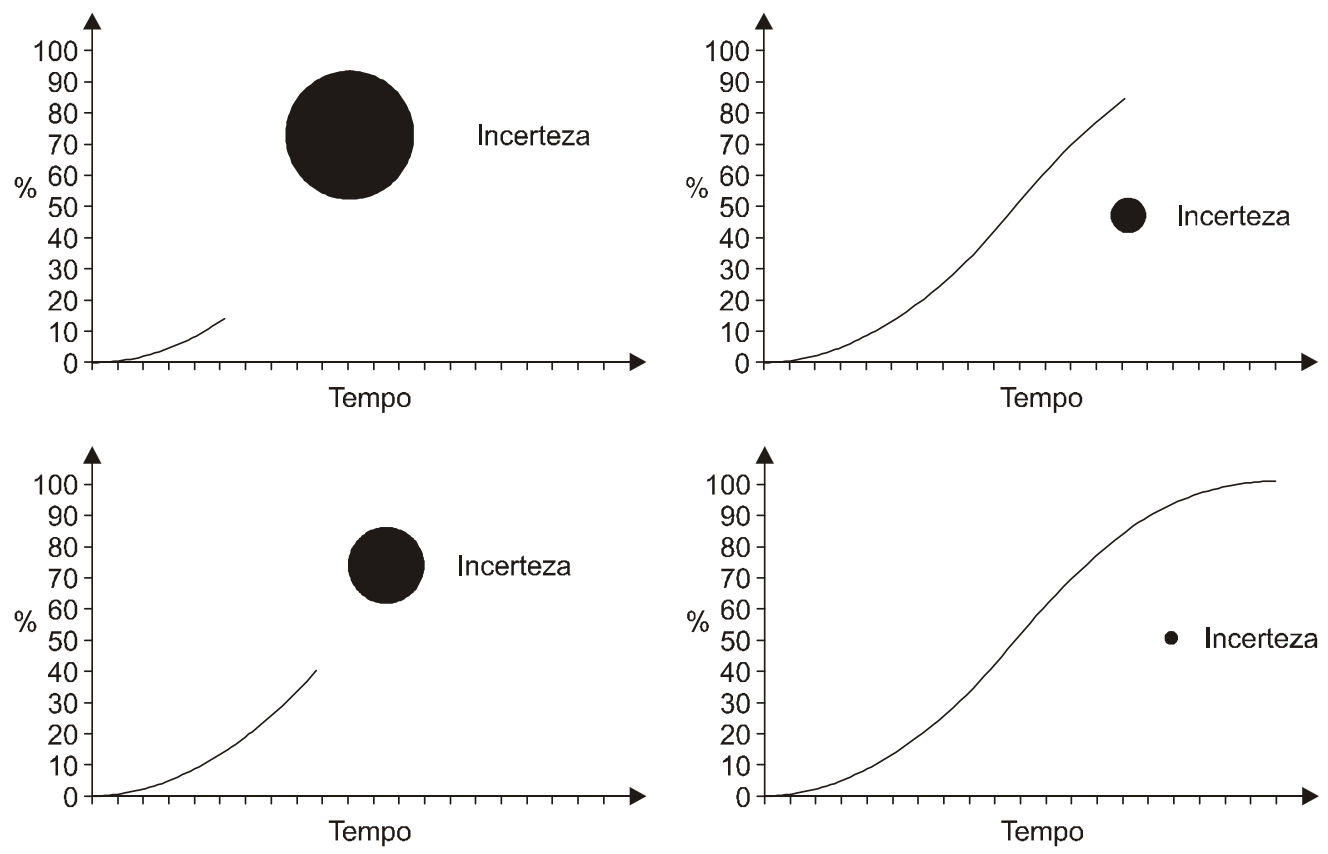

Figura 2 - Incerteza versus fase do projeto (Archibald, 1976). 
Os diferentes tipos de riscos foram mapeados em uma matriz $2 \times 2$ (Figura 3). A primeira dimensão utilizada é a importância percebida do fator, considerando sua probabilidade de ocorrência e seu impacto. A segunda dimensão referese ao poder de controle que o gerente do projeto pode exercer sobre determinado fator.

\subsection{A influência dos projetos em andamento}

Os novos projetos de aplicações de TI concorrem pelos recursos com os projetos de TI já em andamento. Por sua vez, os projetos em andamento têm, em geral, uma incerteza menor do que os projetos propostos (Archibald, 1976).

A atividade de acompanhamento desses projetos pode, e deve, tirar proveito desse fato, a fim de extrair estimativas mais precisas para serem usadas na análise e na composição do portfolio de desenvolvimento. Nessa abordagem, o acompanhamento dos projetos deve considerar apenas os recursos necessários a sua conclusão, desconsiderando o que nele já foi investido.

Isso é importante para evitar alguns tipos de erros comuns em processos de tomada de decisão. Apontam nessa direção Cleland \& Ireland (2002) ao descreverem o uso do Método do Valor Líquido Presente (VLP), já que destacam o fato de que se deve considerar apenas o fluxo de caixa (entradas e saídas) futuro esperado.

Um projeto no qual foi investido grande volume de recursos e no qual se verificou que os objetivos iniciais não mais poderão ser atingidos não deve, necessariamente, ser descontinuado. É preciso avaliar as alternativas para sua conclusão, quanto ainda é necessário para concluí-lo e quais os benefícios que poderão ser auferidos. Nota-se que os recursos necessários podem ser muito menores do que aquilo que já foi investido, e os benefícios agora esperados também poderão ser muito menos ambiciosos do que os iniciais. Contudo, se a relação (medida de alguma forma) entre os recursos ainda necessários para concluir o projeto e os benefícios ainda possíveis se mostrar vantajosa, o projeto não deverá ser interrompido. Vale aqui a idéia de que, quanto mais o projeto avançar no cronograma, maiores serão as razões para ele ser concluído.

Ao desconsiderar, formalmente, o que já foi investido no projeto, diminui a probabilidade de ocorrência da "Síndrome do Jogador", caracterizada por uma atitude do tipo "o dobro ou nada" (Hammond et al., 2001), na decisão de continuidade do projeto. Alguns gerentes, por já terem investido significativamente em algum projeto, sentem-se emocionalmente comprometidos com ele e acreditam que devem "salvar" o projeto, levando-o, a qualquer custo, a atingir seus objetivos iniciais. Fazendo isso, muitas vezes, assumem riscos que normalmente não assumiriam se não estivessem tão envolvidos emocionalmente.

Portanto, os projetos atuais em desenvolvimento competem por recursos com os novos projetos - projetos que estariam em desenvolvimento se houvesse recursos disponíveis, especialmente recursos humanos.

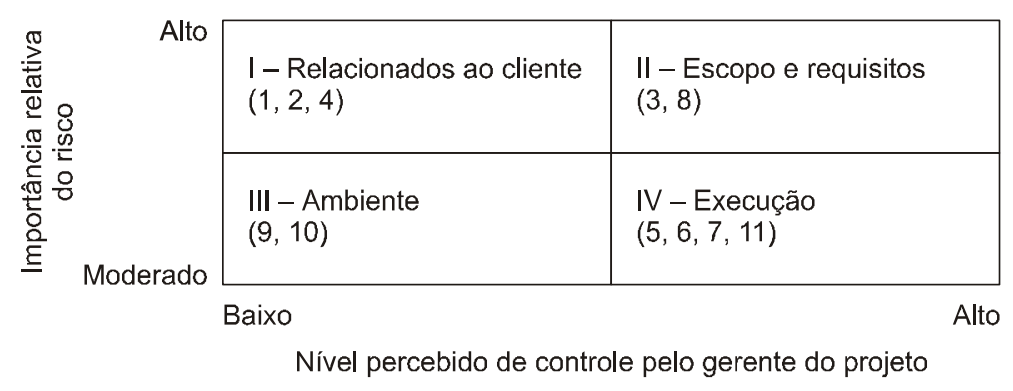

Figura 3 - Fatores de risco (adaptada de Keil et al., 1998). 
O acompanhamento de projeto deve avaliar, a cada instante, se os projetos em desenvolvimento apresentam o alinhamento estratégico desejado, os riscos aceitáveis e o desempenho esperado. No caso de serem observados desvios, deve-se procurar identificar suas causas (Maximiano, 1997). Assim, o processo de acompanhamento (ex cursus) liga-se à avaliação ex ante.

\subsection{Técnicas de acompanhamento de projetos}

Moraes (1999) apresenta uma revisão de técnicas de acompanhamento e controle de projetos na qual são destacadas quatro formas usualmente utilizadas:

- porcentual concluído e duração remanescente;

- curva "S" e análise de tendência;

- valor adquirido (earned value);

- relatório de posição (status report).

A quantificação do porcentual concluído de uma atividade supõe a existência de um critério de medição para a atividade, o que nem sempre é fácil. No caso de um software cujo tamanho estimado é de 5.000 linhas de código, se já tiverem sido escritas 2.500 linhas de código, poderíamos dizer que metade da codificação foi realizada. Contudo, não seria possível afirmar que as 2.500 linhas restantes exigirão o mesmo tempo e esforço (custo) do que aquelas já escritas. Dessa forma, seria mais cauteloso, em vez de medir (ou estimar) o porcentual concluído de uma atividade, trabalhar com a duração remanescente. A duração remanescente, ao se referir à parcela da atividade ainda não executada, é, portanto, mais útil para a avaliação do projeto em andamento do que o porcentual concluído.

A curva " $S$ " é um gráfico que mostra a evolução (porcentual concluído) do projeto no tempo (Figura 4). O projeto começa a ser executado de forma lenta e o ritmo vai aumentando durante a execução, até se aproximar do término, quando volta a cair. A curva "S" mostra a região na qual o projeto não está nem atrasado nem adiantado em relação à data de término (Dinsmore, 1992). Ao fazer o apontamento da execução nesse gráfico, pode-se perceber quando há tendência de atraso antes que ele ocorra de fato. Isso permite a tomada de ações corretivas para adequar o empreendimento a um ritmo esperado. Esse tipo de análise é chamado de análise de tendência. Pela tendência observada, pode-se prever se haverá alguma alteração na data originalmente prevista para o término.

A técnica de controle valor adquirido (earned value) permite comparar o andamento físico do projeto com o andamento financeiro (Figura 5). Por exemplo, um projeto em desenvolvimento que está atrasado, isto é, o andamento físico está abaixo do programado. Se seu custo acumulado até o momento também está abaixo do programado, esse projeto apresenta problema de custo. $\mathrm{O}$ valor adquirido ajuda a considerar simultaneamente aspectos físico e financeiro. $\mathrm{O}$ ' $\Delta$ físico' é a variação porcentual do volume de tarefas entre o programado e o realizado. E o ' $\Delta$ financeiro' é a variação porcentual de custo entre o programado e o realizado. $\mathrm{O}$ valor adquirido é a relação entre ' $\Delta$ físico' e ' $\Delta$ financeiro'. Se seu valor for maior que 1 significa que, independentemente do estágio atual do projeto, ele terá um custo menor do que o previsto. Se for menor, o custo do projeto será maior do que o esperado (Farid \& Karshenas, 1988).

O status report é um procedimento que permite formalizar o estágio de desenvolvimento do projeto em determinado instante no tempo (Figura 6). Esse tipo de relatório deve trazer, em destaque, identificação do projeto: seu nome/ código, objetivo de custo, prazo e desempenho técnico, escopo, nome do gerente/coordenador/ responsável e pessoal-chave.

Eventualmente, também pode conter um breve histórico do projeto até o momento de sua edição. Usualmente, contém a descrição do desempenho do projeto em custo e prazo e nos aspectos técnicos desde a última edição do relatório. Também costuma apresentar a evolução do projeto no período, os problemas encontrados e uma expectativa de desempenho futuro, podendo também conter (não necessariamente) recomendações (Archibald, 1976). 


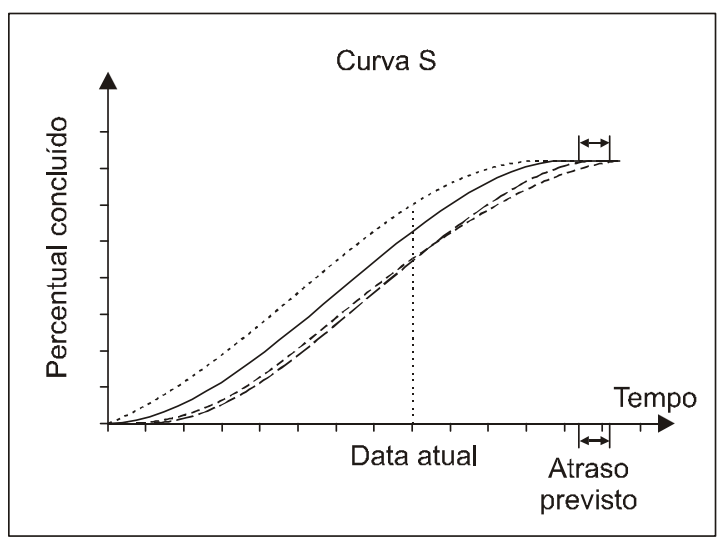

Figura 4 - Curva "S" (Moraes, 1999).

Portanto, considerando esses aspectos, a abordagem de gestão de portfolio adotada neste artigo está baseada nos seguintes conceitos: Fatores Críticos de Sucesso - FCS (Rockart, 1979), posicionamento estratégico (McFarlan, 1984), incertezas dos projetos (Archibald, 1976; Jiang et al., 1996; Jiang \& Klein, 1999b), tipos de projeto (em termos de complexidade e experiência com a tecnologia associada), prazo e orçamento (McFarlan, 1981; Jiang \& Klein, 1999a; Farbey et al., 1995).

\section{Aspectos metodológicos}

$\mathrm{O}$ presente artigo visa a investigar alguns pontos relativos à seleção de projetos de TI: como é feita a seleção de projetos de TI considerando sua ligação e com os objetivos estratégicos da organização, bem como os aspectos dos riscos associados e da utilização de recursos; e como é feita a seleção de projetos de TI caso sejam tratados de acordo com o conceito de portfolio, em vez de cada projeto isoladamente.

Aspectos de funcionalidade dos sistemas associados aos projetos não serão considerados. As decisões de implementação de aplicações de TI podem envolver tanto o desenvolvimento interno como eventual outsourcing. Para esse fim, optou-se por uma pesquisa de caráter explo- ratório, desenvolvidos por intermédio da abordagem de estudo de caso, que permite analisar como os fatores estão envolvidos no processo (Yin, 1991; Claver et al., 2000). A análise dos dados do estudo de caso será feita com base nos conceitos apresentados na seção anterior, que constituem a base teórica do presente artigo.

Os critérios para seleção do caso foram a existência de expressivo e diversificado conjunto de projetos de SI e a presença de um processo formal de gestão de projetos na área de TI.

Com base nesses critérios, a organização selecionada, uma empresa de capital nacional, é uma das maiores e mais importantes da indústria de materiais de construção no Brasil e adota a diferenciação como estratégia de competição. As informações foram obtidas por meio de entrevistas semi-estruturadas realizadas com vários atores de diferentes níveis estratégicos das áreas de negócio e TI.

Após uma "primeira rodada" do estudo de caso, os resultados foram apresentados à empresa estudada. Foram constatadas lacunas no tratamento sistemático da ligação dos projetos com a estratégia de empresa e na gestão de riscos. Também notou-se inexistência de uma abordagem que tratasse do portfolio dos projetos em desenvolvimento. Visando a incluir esses aspectos, uma nova proposta de procedimentos para seleção de projetos de TI foi desenvolvida. 


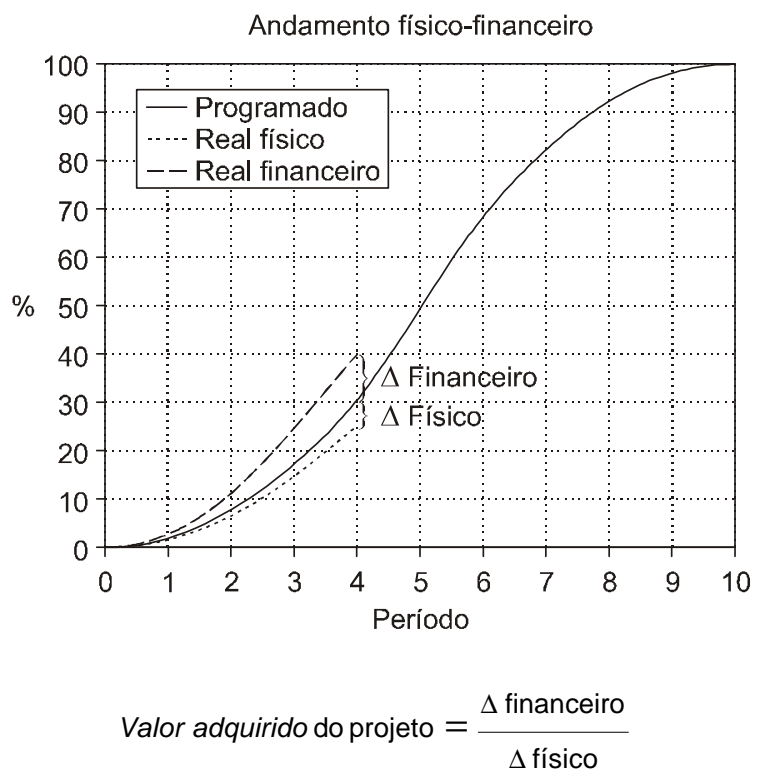

Figura 5 - Exemplo do gráfico de valor adquirido (Moraes, 1999).

Em seguida, aprofundou-se o estudo de caso na mesma empresa, buscando compreender como esses novos procedimentos propostos afetaram a organização.

\section{O estudo de caso}

A organização selecionada, doravante chamada de Empresa 'A', como já destacado, é uma das mais importantes na indústria brasileira de materiais de construção (receita de US\$ 400 milhões por ano e 6 mil funcionários, aproximadamente). Ela está organizada em quatro diferentes unidades estratégicas de negócio, com características operacionais bastante distintas e geograficamente dispersas. Os dados deste estudo foram obtidos por meio de entrevistas semiestruturada realizadas com vários atores de diferentes níveis estratégicos das áreas de negócio e da corporação, envolvendo tanto a área de TI como áreas usuárias. As empresas brasileiras de material de construção têm adotado diferentes estratégias competitivas para responder ao crescente aumento da participação de competidores externos cujos custos são menores. As duas principais abordagens competitivas percebidas são: estratégia de liderança em custos e diferenciação (conforme a classificação de Porter, 1979). No decorrer dos anos, a Empresa 'A' obteve liderança em seus mercados por meio da estratégia de diferenciação (Porter, 1979), uma vez que seus produtos são bem conhecidos pela qualidade e pelo design superiores. Outro importante fator de diferenciação é a assistência técnica, oferecendo serviços de reparos e permitindo que os consumidores comprem, facilmente, peças de reposição.

Considerando esse cenário, e de acordo com os executivos de TI da corporação e das unidades de negócio, os Fatores Críticos de Sucesso (FCS) na Empresa 'A' são: 1. design de novos produtos e prazo de lançamento no mercado; 2. qualidade de processo e do produto final; 3. imagem de qualidade do produto; 4. serviços de pós-venda; 5. custos; 6. flexibilidade e amplitude no mix de produtos; e 7. prazo de entrega dos produtos. 


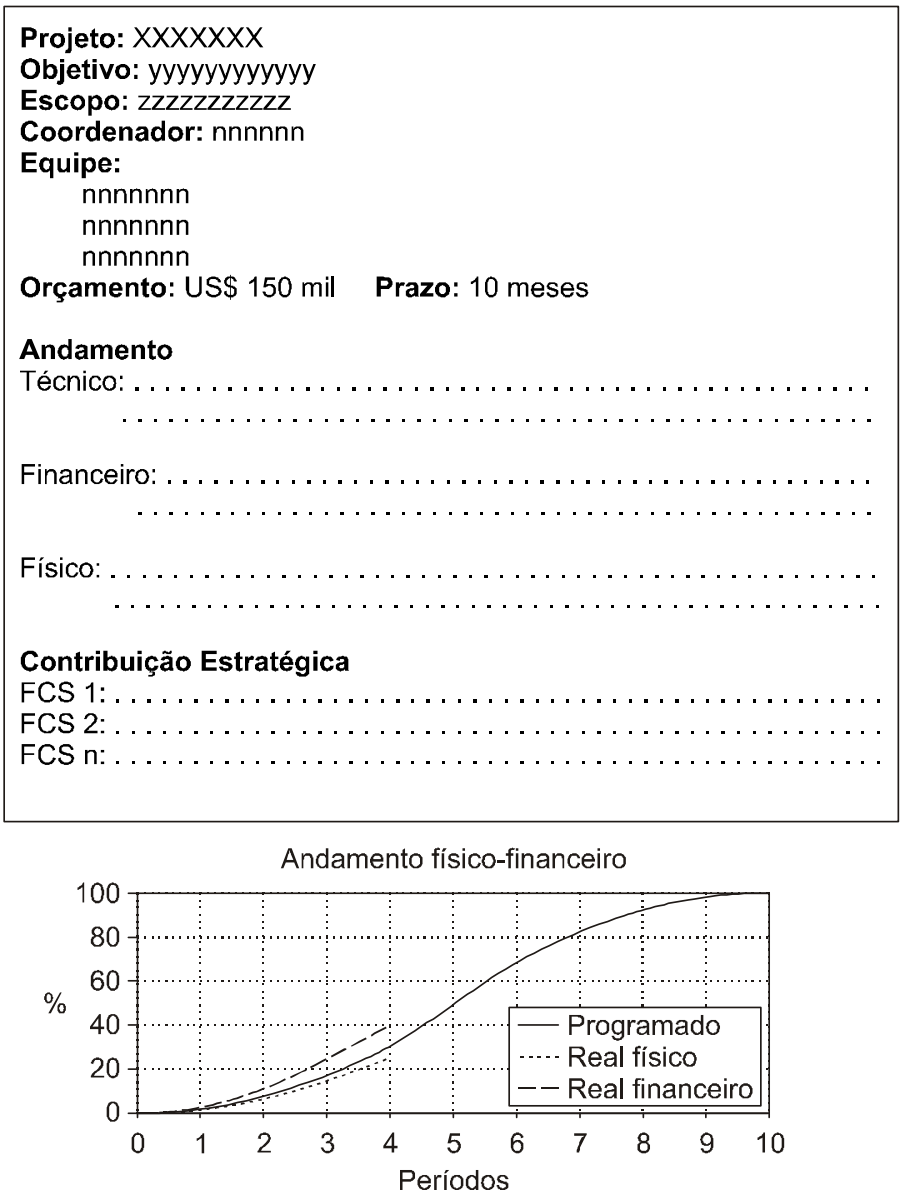

Figura 6 - Exemplo de relatório de projeto (status report).

Nota-se que os quatro primeiros FCS corroboram com o posicionamento estratégico tradicional da empresa que vem garantindo a liderança do mercado, que é o de competir por diferenciação. Por sua vez, os três últimos FCS também apontam para a crescente preocupação com as novas demandas de mercado e com o aumento de competidores, incluindo produtos importados, usualmente de custo mais baixo. A demanda por prazos de entrega curtos também reflete essa situação. A importância dada à flexibilidade e a um mix variado de produtos indica uma nova forma de diferenciação, na qual a empresa tenta usar seus pontos fortes em relação à concorrência. Finalmente, observa-se uma tendência de crescente pressão por características tecnologicamente mais sofisticadas nos produtos, o que se reflete no primeiro FCS.

Nota-se que a empresa ' $A$ ' pode ser considerada uma manufatura tradicional (em termos do uso de TI). Nessas condições, a TI tem papel de suporte e não há perspectivas de mudança desse papel em futuro próximo. As aplicações de TI existentes, bem como aquelas que se planeja implantar a curto prazo, não apresentam impactos estratégicos. Dessa forma, pode ser classificada no quadrante Suporte do Grid Estratégico (Figura 7).

Durante a década de 1990, a Empresa 'A' enfrentou mudanças no grupo da alta gerência. A nova cúpula diretiva iniciou um processo de centralização com uma política de redução de custos nas áreas administrativas, o que levou a 
drástica redução de pessoal na área corporativa de TI (de 200 funcionários, em 1990, para cerca de 40, em 2002). Esses dois aspectos, centralização e controle de custos, são características marcantes da nova cultura da empresa. Todavia, durante quase toda a década de 1980 e começo da de 1990 (apogeu desse modelo), houve um processo de descentralização da TI na empresa (outras áreas da empresa, como suprimentos, também foram descentralizadas), até virtualmente desaparecer nos dias atuais. No período estudado e no âmbito da corporação, a área de TI está subordinada a uma das diretorias executivas corporativas.

$\mathrm{O}$ rol de atribuições das equipes de TI das unidades de negócio foi reduzido ao suporte a usuários finais e, a seguir, aos padrões e às prioridades definidos pela área corporativa de TI. A cultura da organização privilegia o desenvolvimento interno de aplicações, mas isso está mudando lentamente. Alguns pacotes de software têm sido comprados para complementar pontos específicos da arquitetura dos SI. A área corporativa de TI não possui um método sistemático para planejar e controlar seus projetos sob os aspectos de custos e recursos necessários, tampouco para avaliar o desempenho das aplicações. Também não há processo formal ou regular de avaliação ex ante dos projetos de TI, tampouco para avaliar os resultados das decisões sobre a fila de projetos de TI.
Os critérios adotados pelo CIO (Chief Information Officer - principal executivo de TI da empresa) para selecionar os projetos de TI a ser desenvolvidos são: reparo de bugs, modificação de ordem legal, projetos corporativos (diretamente determinados pelo CEO - Chief Executive Office, isto é, principal executivo da empresa) e projetos das unidades de negócio (ao menos um em cada unidade de negócio). Também há "projetos tecnológicos", iniciativas da própria área de TI. Esses projetos envolvem, principalmente, construção e melhoria da infraestrutura em TI.

Não há fórum ou processo formal que permita ao CIO ou a outros gerentes de TI participar diretamente das discussões sobre a estratégia de negócio da organização. Um resumo das características da área corporativa de TI na Empresa 'A' é mostrado na Tabela 1.

\section{Proposta de procedimentos para seleção de projetos de $\mathrm{TI}$}

Os pontos principais de análise do caso apresentado foram discutidos com o CIO e outros gerentes de TI e de áreas de negócio. Com base nessa discussão e na abordagem de gestão de portfolio, um novo conjunto de procedimentos para seleção de projetos de TI foi proposto e analisado na Empresa 'A'.

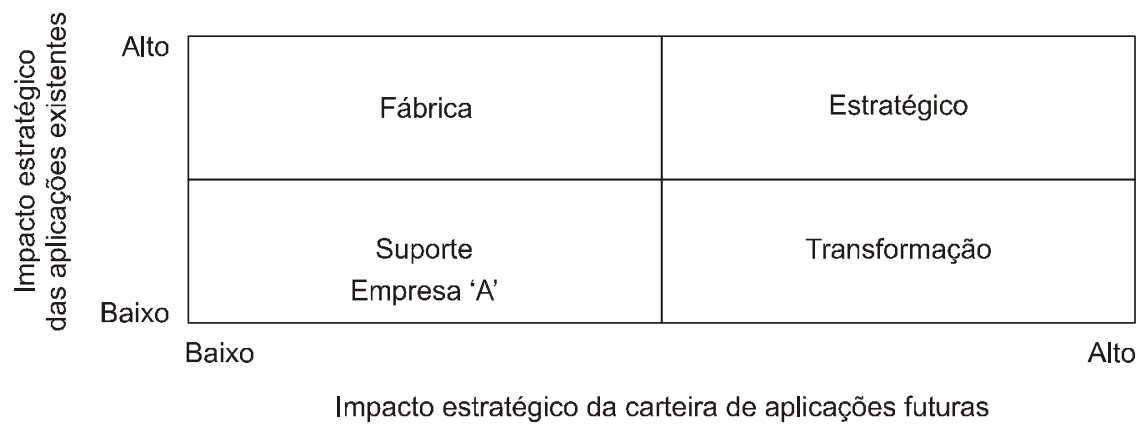

Figura 7 - Enquadramento do estudo de caso no Grid Estratégico (adaptada de McFarlan, 1984). 
Tabela 1 - Organização da área de TI na Empresa 'A'.

\begin{tabular}{lc}
\hline Elemento da TI & Característica da empresa 'A' \\
\hline Estrutura da TI & Por sistemas \\
Operação da TI & Descentralizada \\
Desenvolvimento de sistemas & Centralizada; interna \\
Razões para descentralização da TI & Dispersão geográfica; unidades de negócio; \\
Modelos de eficiência & desejo de controle \\
Controle dos projetos de TI & Nenhum modelo formal \\
\hline
\end{tabular}

Esses procedimentos buscam formalizar o processo de construção da carteira de desenvolvimento usando uma abordagem de gestão de portfolio alinhada à estratégia da empresa e considerando os projetos atualmente em desenvolvimento, já que estes concorrem com os novos no uso dos escassos recursos. O processo de teste desses novos procedimentos foi objeto de aprofundamento do estudo de caso na empresa.

Os procedimentos propostos incluíam os seguintes passos:

- determinação dos FCS (que já havia sido previamente feita);

- classificação dos projetos de TI a desenvolver na empresa, de acordo com suas características e seus impactos na organização como um todo, e na utilização de recursos da equipe de TI;

- formar um portfolio viável de projetos de TI a desenvolver, a partir do conjunto de projetos em carteira;

- estimar os custos de cada um desses projetos de TI do portfolio;

- estimar a probabilidade de sucesso de cada um desses projetos de TI do portfolio;

- estimar a contribuição de cada um desses projetos aos FCS da empresa; e

- escolher o portfolio que proporcione maior contribuição global ao conjunto dos FCS da empresa.

Portanto, inicialmente seria necessário classificar os diferentes tipos de aplicações de TI. Uma vez que a classificação dos oito diferentes tipos de projetos propostos por Farbey et al. (1995) foi considerada muito complexa pelos gerentes de TI da organização estudada, uma nova classificação foi proposta, com apenas quatro diferentes tipos de projetos de TI. São eles:

- obrigatórios: projetos que não possuem alternativas à sua execução. Esse foi o caso, por exemplo, das aplicações que tinham problemas relacionados ao bug do milênio, já que as empresas eram obrigadas a modificar seus SI para evitar sérios problemas futuros. Alterações legais podem ter o mesmo efeito sobre os projetos de SI;

- infra-estrutura: nesse tipo de projeto os benefícios imediatos são pouco significativos, mas criam novas e importantes oportunidades. Exemplos dessa categoria são: implantação de um novo banco de dados corporativo, de uma rede local, implantação de intranets ou uma nova estrutura organizacional;

- incremental: projetos que envolvem tecnologia ou processo de negócio bem conhecido, cujos impactos são facilmente previstos. Trazem ganhos incrementais em eficiência e/ou eficácia;

- exploratórios: projetos que envolvem novas tecnologias (em relação ao conhecimento dominado pela organização), novas estratégias de negócio e/ou novas estruturas/processos organizacionais. São pro- 
jetos de grande incerteza intrínseca, contudo, se forem bem-sucedidos, podem trazer grandes ganhos (em termos de eficiência e/ou eficácia) para a organização.

Conforme convenção adotada pela empresa, considerou-se o prazo de desenvolvimento como o tempo decorrido desde o início formal do projeto até o projeto gerar os benefícios esperados. O orçamento planejado, por sua vez, está relacionado ao investimento necessário para gerar esses benefícios.

A proposta enfatizou a necessidade de avaliação ampla dos projetos, a consideração dos diferentes tipos de projetos e a necessidade de alinhamento estratégico (Henderson \& Venkatraman, 1993).

Não há algoritmo heurístico envolvido. Apesar de esses procedimentos terem permitido a participação de diferentes stakeholders, foi baseado na avaliação subjetiva dos gerentes. Contudo, ele permite que os gerentes reflitam de maneira mais abrangente e qualitativa sobre o escopo e os benefícios para a organização.

Os gerentes (tanto de TI como de negócios) devem procurar selecionar um portfolio de projetos com a melhor combinação de atendimento aos critérios utilizados, que, freqüentemente, podem se tornar conflitantes. Esse processo permite avaliar a importância das diferentes contribuições que a área de TI pode dar à organização.

Os procedimentos propostos procuraram interrelacionar os conceitos previamente apresentados. Uma vez classificados os projetos, o passo seguinte foi listar os projetos candidatos a serem parte da carteira de projetos de TI. O escopo desses projetos pode variar desde a simples manutenção de sistemas existentes ou substituição de hardware até novos e estratégicos SI. Projetos já em desenvolvimento não devem ser interrompidos. Essa restrição está de acordo com a política interna da Empresa 'A' para condução dos projetos de TI. Contudo, conforme já exposto, deve-se ressaltar que essa restrição deveria ser extinta, a fim de haver melhor alocação de recursos.
A primeira lista de projetos de TI, com estimativa de sucesso de cada projeto e sua classificação, é mostrada na Tabela 2. A descrição de cada projeto foi omitida por razões de confidencialidade de informações da Empresa 'A'. Os gerentes de TI estimaram (subjetivamente) o custo e a probabilidade de sucesso de cada projeto.

Foi feita avaliação que atribui uma nota relativa à situação de cada um dos FCS. Essa nota, um número entre 0 (péssimo) e 1 (excelente), é representada por um valor porcentual. Em seguida, para cada um dos projetos propostos foi feita estimativa de sua contribuição para a melhoria de cada um dos FCS, o que permitiu construir a Figura 8, que mostra, portanto, a contribuição estimada (em termos de melhoria, de forma porcentual, dos FCS) de cada projeto em relação a cada FCS. Essas estimativas também foram feitas pelos gerentes de TI. Deve-se notar que cada projeto pode contribuir para mais de um FCS. Os FCS (2) qualidade de processo e do produto final e (5) custos aparentam ser os que sofrerão o maior impacto do conjunto de projetos. Isso é consistente com a tradição gerencial dessa organização. Todavia, esse gráfico não mostra adequadamente o efeito combinado dos projetos sobre os FCS. Para entender os efeitos combinados, o próximo passo foi avaliar o efeito global de cada possível carteira de desenvolvimento. A carteira com a maior contribuição potencial global torna-se, em princípio, forte candidata a ser escolhida. A Figura 9 apresenta o resultado de uma carteira de projetos de TI incluindo todos os projetos da Tabela 2. A área menor (hachurada e mais escura) em torno da grande área central representa os ganhos esperados de uma carteira específica.

Outro importante aspecto a ser considerado na seleção dos projetos é o grau de incerteza gerado pelas características dos projetos e dos recursos alocados em seu desenvolvimento (custo orçado). As incertezas podem ser amenizadas por meio de disponibilidade suficientemente grande de recursos, isto é, por um grande orçamento (por exemplo, se uma quantidade maior de analistas é alocada, a incerteza em relação ao cumprimento de prazos tende a ser reduzida). 
Tabela 2 - Características dos projetos de TI da Empresa 'A'.

\begin{tabular}{cccc}
\hline Projeto & CuSto estimado & Probabilidade de sucesso (incerteza) & Tipo \\
\hline A & USD 210.000 & $90 \%$ & Incremental \\
B & USD 320.000 & $75 \%$ & Incremental \\
C & USD 360.000 & $50 \%$ & Incremental \\
D & USD 250.000 & $90 \%$ & Incremental \\
E & USD 250.000 & $85 \%$ & Incremental \\
F & USD 280.000 & $60 \%$ & Exploratório \\
G & USD 250.000 & $85 \%$ & Obrigatório \\
H & USD 180.000 & $50 \%$ & Exploratório \\
I & USD 210.000 & $40 \%$ & Exploratório \\
J & USD 320.000 & $60 \%$ & Exploratório \\
K & USD 360.000 & $75 \%$ & Incremental \\
L & USD 280.000 & $70 \%$ & Incremental \\
M & USD 140.000 & $95 \%$ & Infra-estrutra \\
N & USD 70.000 & $97 \%$ & Infra-estrutra \\
O & USD 30.000 & $92 \%$ & Infra-estrutra \\
P & USD 100.000 & $93 \%$ & Infra-estrutra \\
Q & USD 70.000 & $98 \%$ & Infra-estrutra \\
R & USD 250.000 & $70 \%$ & Infra-estrutra \\
\hline & & &
\end{tabular}

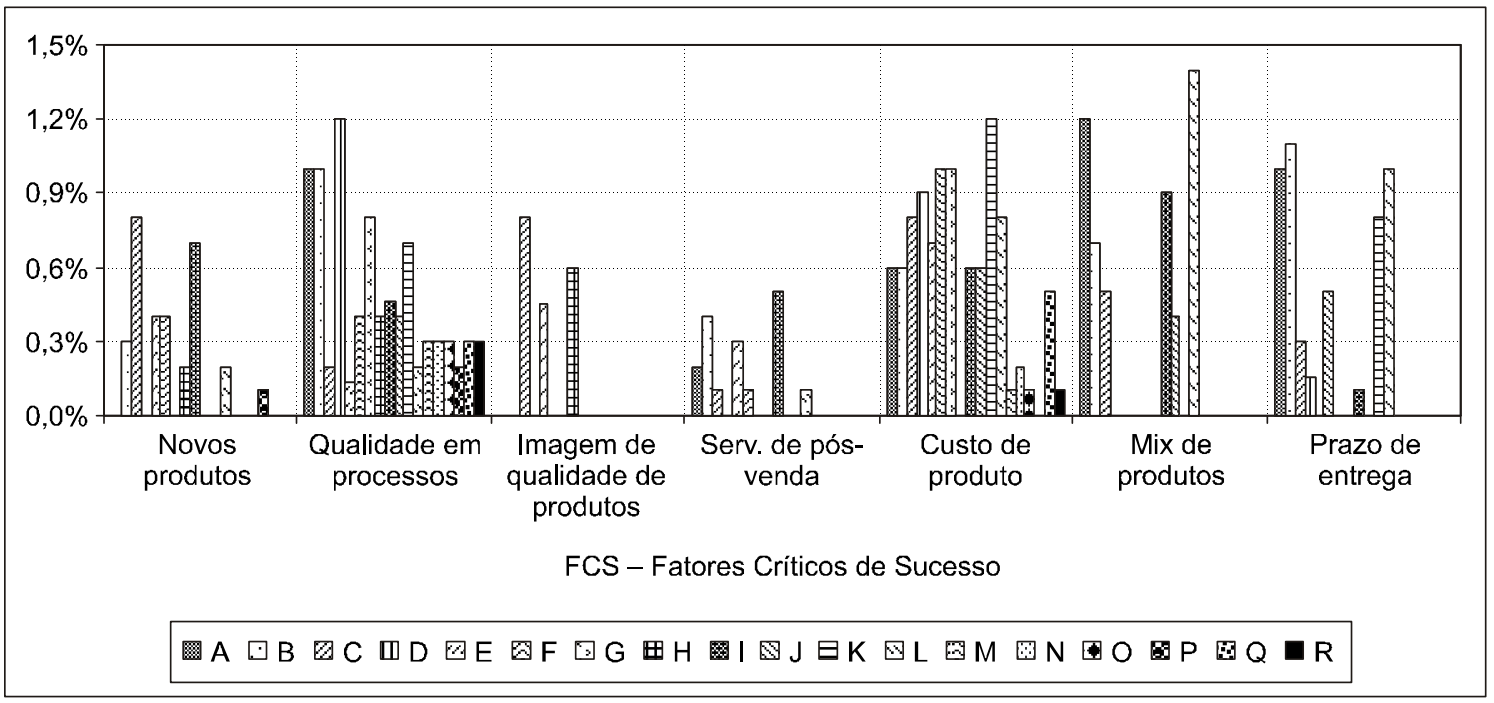

Figura 8 - Contribuição estimada dos projetos de TI para cada FCS. 
A probabilidade de sucesso (relacionada a essas incertezas) dos projetos está associada à classificação apresentada anteriormente. Assim, cada projeto foi classificado e sua incerteza foi estimada, como já mencionado. Na Figura 10, os projetos são classificados de acordo com os quatro tipos propostos (obrigatório, infra-estrutura, incremental e exploratório) e cada projeto é representado por um círculo, cuja área representa o investimento necessário.

Esse gráfico permite avaliar investimentos de grande incerteza. Uma importante pergunta que poderia ser feita é: devem-se alocar recursos escassos em projetos de grande incerteza? Os projetos ' $\mathrm{C}$ ' e 'I', cujos investimentos representam, respectivamente, US\$ 360 mil e US\$ 210 mil e as probabilidades de sucesso estimadas em $50 \%$ e $40 \%$, poderiam representar essa situação. Por outro lado, se esses projetos são considerados importantes por outros critérios, devem receber mais recursos ou ter seu escopo revisto de forma a poderem atingir seus objetivos com menor incerteza. Da mesma maneira, projetos obrigatórios não devem apresentar grade incerteza, já que o fracasso traz graves consequiências. Os trade-offs desse processo refletem a competição associada à estrutura da indústria na qual a empresa atua, bem como as competências internas da empresa.

\section{Conclusões}

$\mathrm{O}$ estudo de caso mostrou alguns dos problemas enfrentados pelos gerentes de TI em busca de melhor alocação de recursos entre vários projetos. Ele levou a proposta de um conjunto de procedimentos abrangente (que considera questões de eficácia, eficiência e risco) e apresentou potencial para ser aplicado nas organizações. Usualmente, as equipes de TI focam muito a busca do sucesso técnico em seus projetos, bem como as funcionalidades presentes nas aplicações desenvolvidas. Com a abordagem de gestão por portfolio proposta e estudada, as preocupações de natureza gerencial e mesmo estratégica ficam presentes no processo de planejamento e controle desses projetos. Um dos pontos a ser melhor analisado é a classificação dos projetos de TI, uma vez que a tipologia adotada reflete a situação específica da empresa estudada e o ponto de vista de seus gerentes de negócios e de TI. A eficiência e a eficácia da gestão dos projetos em desenvolvimento não foram analisadas, tampouco o resultado dos projetos já concluídos.

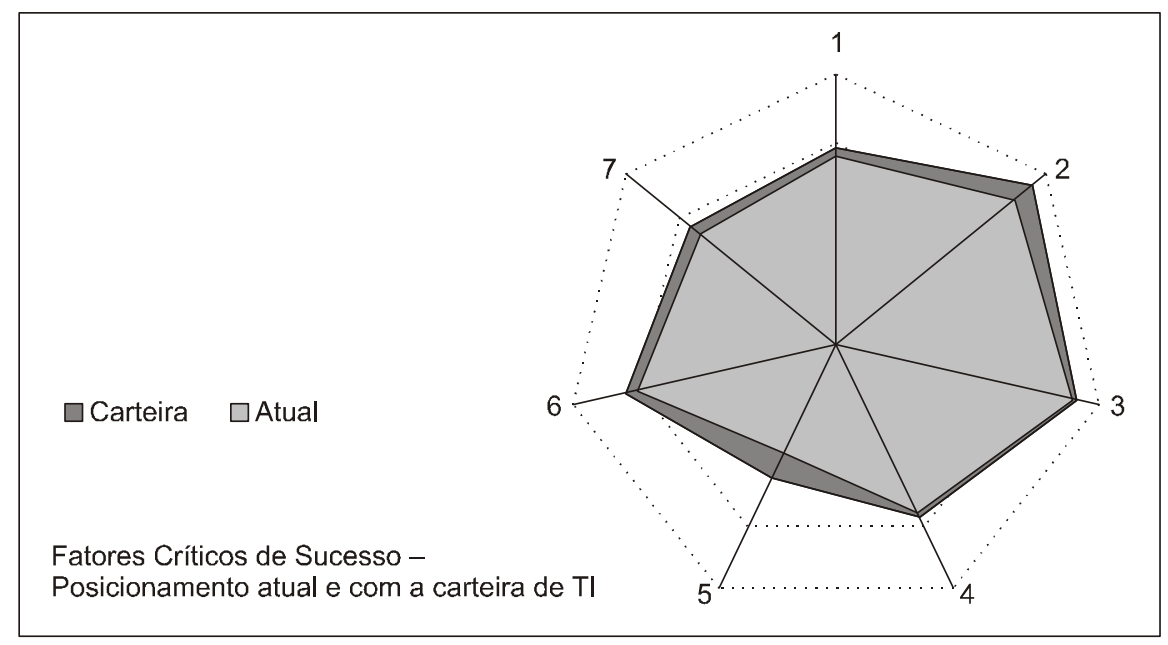

Figura 9 - Melhoria da eficácia pela carteira de projetos em relação aos FCS. 


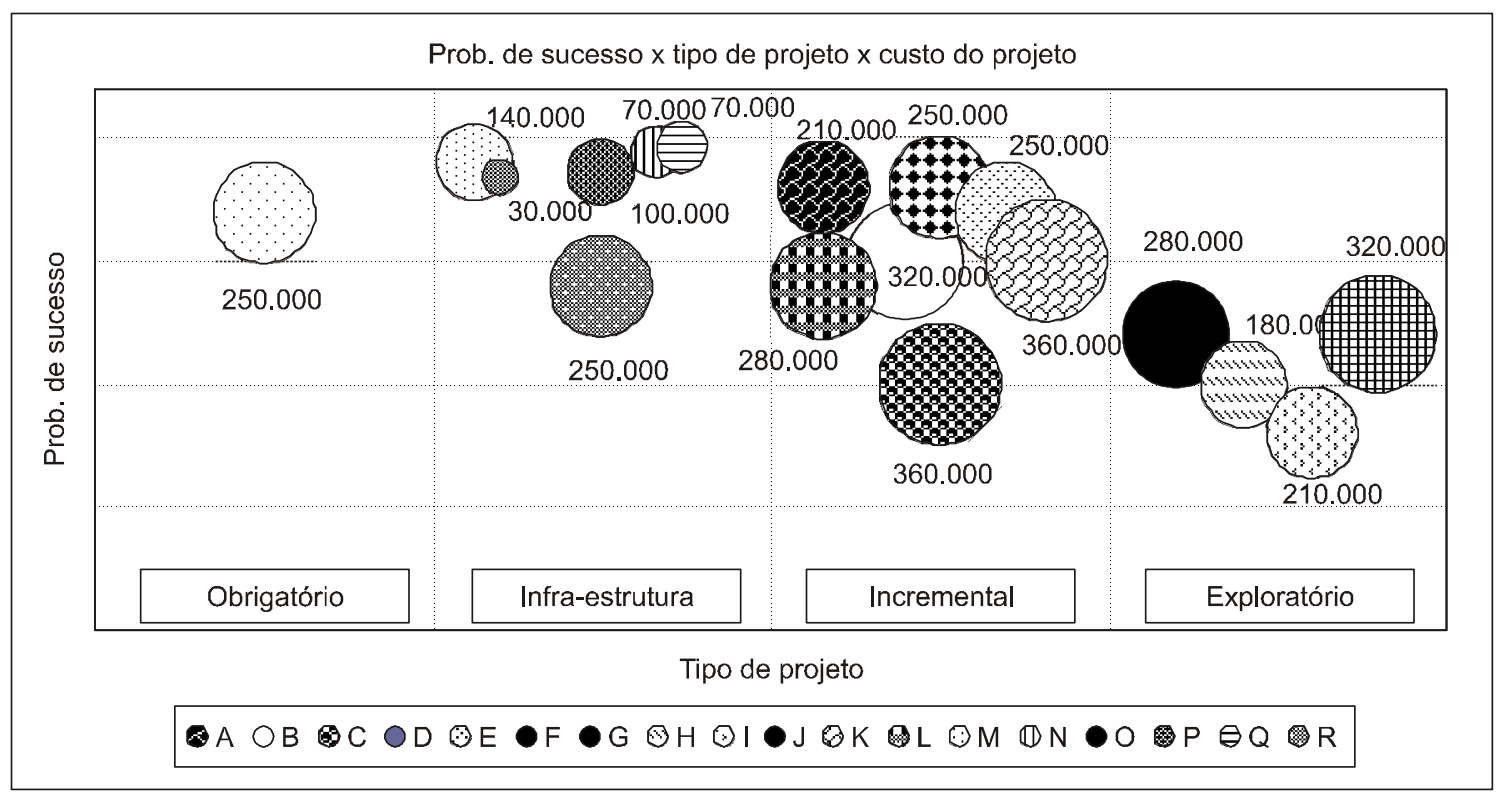

Figura 10 - Incertezas dos projetos de TI, classificados em quatro tipos.

Essas duas questões devem ser mais bem estudadas em novas pesquisas. Algumas das dificuldades no uso da proposta foram causadas pelo fato de ser a primeira vez que os gerentes participaram do processo e não tinham familiaridade com os conceitos utilizados.

Uma vez que múltiplos objetivos devem ser satisfeitos pelo processo de seleção da carteira de projetos de TI, algumas técnicas poderiam ser de grande valia, como Analytic Hierarchy Process (AHP) ou Fuzzy Sets (Saaty, 1991; Shimizu et al., 2001). Elas poderiam ser utilizadas, por exemplo, na avaliação e na priorização dos efeitos da TI sobre os FCS. Outro ponto passível de melhoria reside no cálculo do risco de cada projeto, que, no caso em questão, foi estimado de maneira subjetiva. Finalmente, o impacto de cada aplicação em cada FCS também poderia ser estimado de maneira menos subjetiva.

Um importante benefício da sistemática proposta foi auxiliar os gerentes a sistematizar suas idéias e refletir sobre o processo e os impactos dos projetos de TI selecionados naquilo que é importante para a empresa. Também permitiu que os profissionais de TI e de outras áreas trabalhassem juntos e, conseqüentemente, entendessem melhor as necessidades das partes envolvidas, o que possibilitou aumentar o tão desejado alinhamento estratégico.

Dessa forma, fica facilitada uma visão estratégica tanto do negócio como da TI, de forma a analisar e avaliar os ganhos na produtividade e na competitividade dos negócios oriundos de maior eficácia no uso da TI, em alinhamento com a operação e a estratégia da empresa. Contudo, ainda persiste a necessidade de eficiência na operação da TI, bem como a importância da capacitação técnica nos processos de gestão de projetos de TI (Carvalho et al., 2003; Laurindo et al., 2002).

Embora a Empresa 'A' deva manter a TI no quadrante Suporte, isso não significa que ela possa negligenciar uma gestão mais integrada entre TI e negócios, incluindo a corporação e as áreas de negócio. Todavia, o fato de os procedimentos de avaliação de eficácia serem pouco elaborados e de os procedimentos de avaliação de eficiência não serem rotineiros está de acordo com o papel de apoio da TI à operação. Finalmente, este artigo pode ser o início de futuras 
pesquisas que deverão tratar das possibilidades de melhoria mencionadas anteriormente, bem como possibilitar o aprofundamento da discussão acerca da gestão de projetos de TI. Entre elas, a análise e a avaliação ex post dos projetos de TI; e a influência do grau de maturidade da organização no processo de seleção e avaliação de seus projetos de TI.

\section{Referências Bibliográficas}

ARCHIBALD, R. D. Managing high technology programs and projects. New York: John Willey, 1976. $278 \mathrm{p}$.

BRYNJOLFSSON, E.; HITT, L. M. Beyond the productivity paradox. Communications of the ACM, Aug. 1998.

BRYNJOLFSSON, E. The productivity paradox of Information Technology. Communications of the $A C M$, v. 36, n. 12, p. 67-77, Dec. 1993.

CARVALHO, M. M.; LAURINDO, F. J. B.; PESSÔA, M. S. P. Information Technology Project management to achieve efficiency in Brazilian Companies. In: KAMEL, S. (Org.). Managing globally with Information Technology. Hershey, 2003. p. 260-271.

CLAVER, E.; GONZALEZ, R.; LLOPIS, J. An analysis of research in information systems (19811997). Information \& Management, v. 37, n. 4, p. 181-195, Apr. 2000.

CLELAND, D. I.; IRELAND, L. R. Gerência de projetos. Rio de Janeiro: Reichmann \& Affonso Editores, 2002.

DINSMORE, P. C. Gerência de programas $e$ projetos. São Paulo: Editora Pini, 1992.

DUHAN, S.; LEVY, M.; POWELL, P. Information Systems strategies in knowledge-based SME's: the role of core competencies. European Journal of Information Systems, v. 10, n. 1, p. 25-40, 2001.

FARBEY, B.; LAND, F. F.; TARGETT, D. A taxonomy of information systems applications: the benefits evaluation ladder. European Journal of Information Systems, v. 4, n. 1, p. 41-50, 1995.

FARID, F; KARSHENAS, S. Cost/schedule control systems criteria under inflatio. Project Management Journal, v. 19, n. 5, Nov. 1988.

HAMMOOND, J. S.; KEENEY, R. L.; RAIFFA, H. As armadilhas ocultas na tomada de decisão. In: RIECH, E. (Trad.). Tomada de decisão. Rio de Janeiro: Ed. Campus, 2001.
HENDERSON, J. C.; VENKATRAMAN, N. Strategic alignment: leveraging information technology or transforming organizations. IBM Systems Journal, v. 32, n. 1, p. 4-16, 1993.

JIANG, J. J.; KLEIN, G. Project selection criteria by strategic orientation. Information \& Management, v. 36, p. 63-75, 1999.

JIANG, J. J.; KLEIN, G. Risks to different aspects of systems success. Information \& Management, v. 36, p. 263-272, 1999.

JIANG, J. J.; KLEIN, G.; BALLOUN, J. Ranking of system implementation success factors, Project Management Journal, v. 27, n. 4, p. 50-55, 1996.

KEIL, M.; CULE, P. E.; LYYTINEN, K.; SCHMIDT, R. C. A framework for identifying software project Risks. Comunications of the ACM, v. 41, Nov. 1998.

LAURINDO, F. J. B.; CARVALHO, M. M.; SHIMIZU, T. Information technology strategy alignment: Brazilian cases. In: KANGAS, K. (Org.). Business strategies for Information Technology management. Hershey, 2003. p. 186-199.

LAURINDO, F. J. B. Tecnologia da Informação: eficácia nas organizações. São Paulo: Editora Futura, 2002. 248 p.

LAURINDO, F. J. B.; CARVALHO, M. M.; PESSÔA, M. S. P.; SHIMIZU, T. Selecionando uma aplicação de Tecnologia da Informação com enfoque na eficácia: um estudo de caso de um sistema para PCP. Revista G\&P: Gestão e Produção, v. 9, n. 3, p. 377-396, São Carlos, dez. 2002.

LAURINDO, F. J. B.; SHIMIZU, T.; CARVALHO, M. M.; RABECHINI Jr., R. O papel da Tecnologia da Informação (TI) na estratégia das organizações. Revista G\&P: Gestão e Produção, v. 8, n. 2, p. 160-179, São Carlos, ago. 2001.

MAXIMIANO, A. C. A. Administração de projetos: como transformar idéias em resultados. São Paulo: Editora Atlas, 1997. 
McFARLAN, W. E. Information Technology changes the way you compete. Harvard Business Review, v. 62, n. 3, p. 98-103, May/Jun. 1984.

McFARLAN, W. E. Portfolio approach to information systems. Harvard Business Review, v. 59, n. 5, p. 142-150, Sep./Oct. 1981.

MORAES, R. O. Planejamento, programação e controle de projetos de software. 1999. Dissertação (Mestrado) - Universidade Paulista, São Paulo.

PORTER, M. E. How Competitive forces shape strategy. Harvard Business Review, p. 137-145, Nov./Dec., 1979.

PORTER, M. E. Strategy and the internet. Harvard Business Review, p. 63-78, Mar. 2001.

PORTER, M. E. What is strategy? Harvard Business Review, p. 61-78, Nov./Dec. 1996.

PORTER, M. E.; MILLAR, V. E. How information gives you competitive advantage. Harvard Business Review, v. 63, n. 4, p. 149-160, July/Aug. 1985.
ROCKART, J. F. Chief Executives define their own data needs. Harvard Business Review, v. 57, n. 2, p. 81-92, Mar./Apr. 1979.

SAATY, T. S. Método de análise hierárquica. São Paulo: McGraw-Hill, Makron, 1991.

SHIMIZU, T.; LAURINDO, F. J. B.; MORITA, H. Decision-making process in organizations: a complex problem. In: CONFERENCE OF THE PRODUCTION AND OPERATIONS MANAGEMENT SOCIETY, 2001, Guarujá, SP. Proceedings... Guarujá, SP, ago. 2001. p. 290-297.

STRASSMAN, P. A. The business value of computers. New Canaan: The information Economic Press, 1990.

STRATOPOULOS, T.; DEHNING, B. Does successful investment in IT solve the productivity paradox? Information \& Management, v. 38, p. 103-117, 2000.

YIN, R. K. Case study research: design and methods. Newbury Park: Rev. Ed. Sage Publications, 1991.

\title{
SELECTING AN INFORMATION TECHNOLOGY APPLICATION THROUGH AN EFFECTIVENESS APPROACH: A PRODUCTION PLANNING SYSTEM CASE STUDY
}

\begin{abstract}
The role of information technology (IT) in organisations can vary from simple administrative support till a strategic position, in which IT achieve an important status in leading companies in increasingly competitive markets. On the other hand, there is still an important discussion about the difficulties of finding evidence of returns over investments in IT. Although there are different explanations for this fact, there is a general consensus about the relevant increase in IT investments in the last decades. The decisions about which IT projects should be implemented are frequently determinant of business performance and are able not only of changing the competitive positioning of the companies but also can modify the competitive structure of industries. This paper aims to analyse, through a case study, the selection of IT projects through a management of portfolio approach, considering both efficiency (use of resources) and effectiveness (achieving positive results for the organisation) issues, and also analysing their risks.
\end{abstract}

Key words: information technology, project portfolio management, ex ante evaluation. 